\title{
Theoretical Descriptions of the Nucleon Electromagnetic Form Factors in the Space- and Time-like regions
}

\author{
Giovanni Salmè \\ ${ }^{a}$ Istituto Nazionale di Fisica Nucleare - Sezione di Roma \\ P.le A. Moro 2, 00185 Rome, Italy
}

Some recent advances in the theoretical description of the Nucleon electromagnetic form factors, both in the space- and time-like regions, will be briefly illustrated. In particular, both the present stage of the lattice calculations and updated phenomenological approaches, like the ones based on dispersion relations and on microscopical models, will be reported.

\section{Introduction}

The Nucleon electromagnetic form factors (em ff's) represent a relevant chapter in our path towards the understanding of hadron dynamics. It is striking that, after almost half a century of experimental investigations, this field could still hold a number of surprises (e.g., the intriguing fall-off of the proton charge ff more rapid than the assumed dipole one), or have some kinematical regions not fully explored (e.g., the timelike region, in order to obtain separate information on ff's ). In particular, let us remind (see, e.g., 112 for recent reviews) that in the spacelike (SL) region the existence of a possible zero in $G_{E}^{p}$ for $-q^{2}=Q^{2}>7 \mathrm{GeV}^{2}\left(q^{2}=\right.$ the square momentum transfer), as suggested by a linear extrapolation of the data obtained by the polarization transfer experiments, could open new perspectives. Indeed, the dipole form of the $\mathrm{Nu}$ cleon ff's is supported by the pQCD, therefore a sharp difference between the charge distribution of the current quarks and the charge distribution of the "constituent" quarks appears a clear signature of NpQCD effects in act. Even low- $Q^{2}$ ff's (cf the forthcoming analysis of E08007 at TJLAB) could give new stringent constraints for builders of phenomenological models. In the the timelike (TL) region, analogously relevant issues can be met. For instance, the neutron data around $q^{2} \sim 4 \mathrm{GeV}^{2}$ show a behavior, $\left|G_{\text {eff }}^{n}\right| \sim\left|G_{\text {eff }}^{p}\right|$, sharply different from the one suggested by pQCD, naively indicating $\left|G_{e f f}^{n}\right| \sim\left|e_{d} / e_{u}\right|\left|G_{e f f}^{p}\right|$. Moreover, TL proton data show many interesting structures (see, e.g. [3]), that could give a lot of information on the hadronic component of the photon wave function.

On the theoretical side, in a very schematic way, one can list the following approaches: i) Lattice calculations, based on a Euclidean 4D space, that can address the SL region only; ii) analytic approaches, based on dispersion relations, that can investigate both SL and TL regions, even yielding predictions for the unphysical interval $0<q^{2}<4 M_{N}^{2}$; iii) purely phenomenological methods, with constituent quarks (CQ) as basic ingredients, that can be exploited for describing both SL and TL regions; iv) field theoretical approaches, like the ones based on the DysonSchwinger equation, that can be applied to SL and TL regions. In this short review we will restrict the presentation to i), ii) and iii) (see, e.g., [1 for a different discussion from i) to iv)). It should be also mentioned a quite recent approach based on the gravity dual models of QCD (or holographic QCD), like the one in Ref. [4].

\section{Lattice "Data"}

In principle, Lattice QCD allows one to achieve a non perturbative description of the Nucleon ff's, as shown by the more and more accurate results obtained in recent years. In particular, such interesting outcomes have taken profit from: i) an im- 
pressive growth of the available computing power, that has allowed in nowadays results, e.g., to release the quenched approximation (where the seaquark loop contributions are disregarded); ii) new algorithms, leading to a better description of the fundamental symmetries of $\mathrm{QCD}$, and last, but not least, iii) substantial improvements of the chiral extrapolation techniques.

On the other hand, we should mention the main obstacles to be overcome:

- in a 4D Euclidean Lattice (i.e., it $\rightarrow t$ ), one cannot directly evaluate the matrix elements of the em current operator. Therefore a careful study of the proper ratio between three- and two-point functions is necessary in order to get "large values" of the Euclidean time, that eventually allows to filter the contribution from hadronic states with Nucleon quantum numbers. This filtering prevents the study of TL ff's, since an exponential fall-off in time lacks for $q^{2}>0$;

- present Lattice data are obtained with values of the coupling constant $\beta=6 / g^{2}$ and the hopping parameter, $\kappa$, that lead to sizable $u, d$ quark masses. Usually, the calculations are labeled by the corresponding pion mass, from which one can infer the quark mass in the actual data. In order to move toward the current quark masses, one has to reduce the Lattice spacing (that allows to accommodate lighter quarks) or to exploit extrapolation formulas, obtained from the low-energy regime of the QCD. Unfortunately such chiral extrapolations have a well-known "non analytic" $\left(\log m_{\pi}^{2}\right)$ behavior for small value of the pion mass. The lowest value of the pion mass in the present Lattice calculations for the Nucleon ff's is $\sim 360 \mathrm{MeV}$ [5];

- the smallness of momentum transfers investigated in a Lattice is related to the space extent, $L$, namely $|\vec{q}| \sim 2 \pi / L$. This has influence on the range of applicability of the chiral extrapolations, about $Q^{2} \leq 0.4 \mathrm{GeV}^{2}$, not always reached by the present Lattices (e.g., $Q_{\text {low }}^{2} \sim 0.23 \mathrm{GeV}^{2}$ for
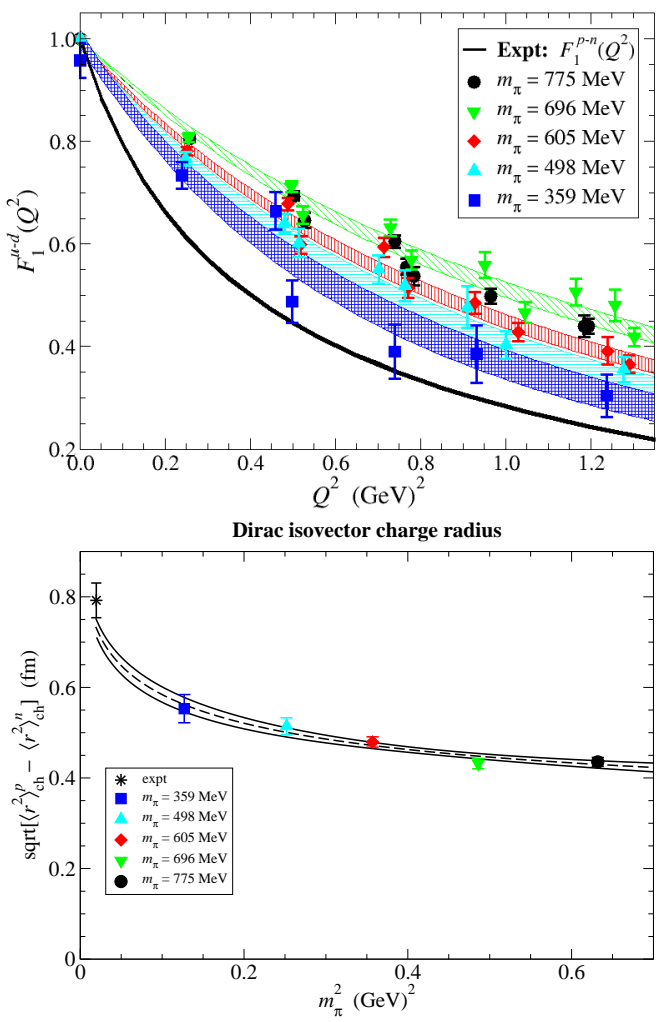

Figure 1. LHP Coll. results for the "isovector" (see text) approximation of the Dirac ff $F_{1}\left(Q^{2}\right)$ (upper panel) and for the "isovector" radius (lower panel). Solid and dashed lines: different extrapolation formulas. (After Ref. [5).

the LHP Coll. [5] and, $Q_{\text {low }}^{2} \sim 0.4 \mathrm{GeV}^{2}$ for the QCDSF Coll. [6] and $Q_{\text {low }}^{2} \sim$ $0.15 \mathrm{GeV}^{2}$, for the Cyprus-MIT Coll. [7, but with quenching approximation);

- "large" momentum transfers in the range of few $\mathrm{GeV}^{2}$ are in principle accessible by nowadays Lattice spacing, but Fourier transforms of two- and three-point functions become noise-dominated for square momentum transfers beyond $2 \mathrm{GeV}^{2}$;

As an example of an updated Lattice calculation of the Nucleon ff's, the results obtained 
by the LHP Coll. [5], for both the "isovector" Dirac ff (obtained through $\langle$ proton $| \bar{u} \gamma^{\mu} u-$ $\bar{d} \gamma^{\mu} d \mid$ proton $\rangle$ ) and the "isovector" radius (with different extrapolations), are shown in Fig. 1 .

\section{Phenomenology I: Dispersion Relations}

Dispersion relation (DR) approaches have a very long story. Within such a framework, one can relate real and imaginary parts of the em ff's, once the unitarity (probability conservation) and analyticity (causality) properties are implemented. The analysis based on DR allows one to investigate both SL and TL regions, and even the unphysical region in the TL region, where a possible bound state $p \bar{p}$ is present, close to the threshold, $q^{2}=4 M_{N}^{2}$. The main ingredient is the DR given by $(i=1,2)$

$$
F_{i}\left(q^{2}\right)=\frac{1}{\pi} \int_{q_{0}^{2}}^{\infty} \frac{\operatorname{Im} F_{i}\left(q^{\prime 2}\right)}{q^{\prime 2}-q^{2}-i \varepsilon} d q^{\prime 2}
$$

or a once subtracted version, where $F_{i}(0)$ (if finite) appears

$$
F_{i}\left(q^{2}\right)=F_{i}(0)+\frac{q^{2}}{\pi} \int_{q_{0}^{2}}^{\infty} \frac{\operatorname{Im} F_{i}\left(q^{\prime 2}\right)}{q^{\prime 2}\left(q^{\prime 2}-q^{2}-i \varepsilon\right)} d q^{\prime 2}
$$

The next ingredient is a suitable Ansatz, physically motivated, for $\operatorname{Im} F\left(t^{\prime}\right)$. For instance, the Bonn group [8] has exploited i) the form suggested by the decay processes $\rho \rightarrow \pi \pi, \phi \rightarrow K \bar{K}$, $\omega \rightarrow \rho \pi$, and related continua and ii) the constrains from $\mathrm{pQCD}$. In this case, the fit to the experimental data (SL nucleon ff's and TL proton data), has 17 free parameters and a total $\chi^{2} / D O F$ of 1.8 . It should be pointed out that the TL neutron calculation represents a prediction of the model. In Fig. 2, the TL Nucleon ff's obtained in the so-called super convergent approach (imposed by a proper fall-off of $F_{i}$ ) are shown.

In Ref. 9] a different approach has been followed, by directly applying DR to the ratio $R\left(q^{2}\right)=\mu_{p} G_{E}^{p}\left(q^{2}\right) / G_{M}^{p}\left(q^{2}\right)$, both in SL and TL regions. A very general form for $\operatorname{Im} R\left(q^{2}\right)$, with six parameters, has been adopted, obtaining a $\chi^{2} / D O F \sim=1.3$. In Fig. 3, the SL and TL ratios are shown (for an analysis of all the ff's see Ref. [11]). The approach yields two in-
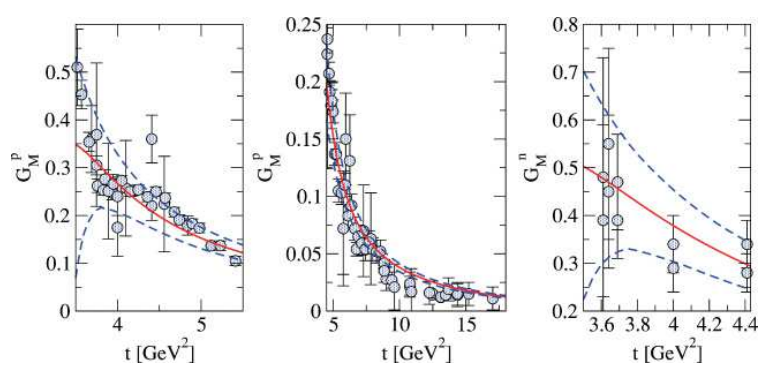

Figure 2. Nucleon ff's for TL momentum transfers. (After Ref. [8])
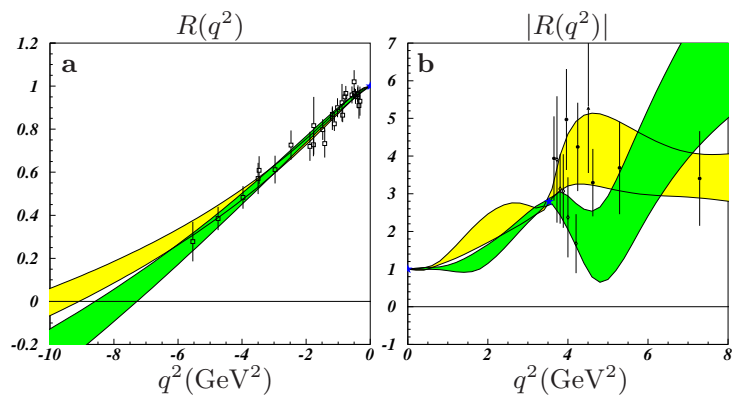

Figure 3. $\quad R\left(q^{2}\right)$ (see text) vs $q^{2}$. The bands represent the error. Green band: retaining in the fitting procedure the TL LEAR data [10. Yellow band: the same as the green band, but retaining the TL BABAR data [3]. (After Ref. [9]).

teresting predictions: i) the position of the SL zero, that depends upon the TL data chosen in the fit, i.e. $q_{z}^{2}(B A B A R)=-10 \pm 1 \mathrm{GeV}^{2}$, $q_{z}^{2}(L E A R)=-7.9 \pm 0.7 \mathrm{GeV}^{2} ;$ ii) the asymptotic limits, extracted from the theoretical expressions

$$
\lim _{q^{2} \rightarrow \pm \infty}\left|\frac{G_{E}^{p}\left(q^{2}\right)}{G_{M}^{p}\left(q^{2}\right)}\right|=\left\{\begin{array}{c}
0.95 \pm 0.20 \text { BABAR } \\
2.3 \pm 0.7 \text { LEAR }
\end{array}\right.
$$

\section{Phenomenology II: Microscopic Ap- proaches}

Relativistic CQ models have been widely adopted for obtaining a microscopic description of the em properties of the Nucleon. Since '90, it 
was recognized the relevance of dressing the CQ's, e.g. by suitable ff's, with a spatial extension of the order of $0.4-0.5 \mathrm{fm}$, (a scale different from a naive pion-cloud model), in order to obtain a very accurate description of the Nucleon and Pion ff's in the SL region (see, e.g., [12]). After that, new approaches, aimed at a more deep understanding of the degrees of freedom beyond the CQ ones, have been developed.

Recently, within the manifestly covariant spectator model, a very accurate description of the Nucleon ff's in the SL region has been achieved [13. The Nucleon, composed of three valence CQ's with form factors, has a center of mass motion described by a solution of the Dirac equation, while the intrinsic dynamics is given by a phenomenological S-wave state, consistent with the properly symmetrized covariant spectator formalism. The independence of the intrinsic wave function upon the direction of the relative momentum of an off-mass-shell quark with respect to an on-mass-shell diquark allows one to construct an internal pure S-wave Nucleon state, and therefore to investigate the Nucleon ff's for a Nucleon living in a well defined orbital angular momentum state. It is worth noting that the free propagation of three quarks is prevented by a suitable choice of the intrinsic wave function. The model has been further improved by adding a two-pion cut for a better description of the ff's at low $Q^{2}$. In Fig. 4, the Nucleon ff's, obtained through fitting procedures that take into account the DIS as well, are shown.

Within the Hamiltonian Light-front dynamics, where a meaningful Fock expansion can be considered, the Pavia group has devised a CQM + "meson-cloud" approach (i.e., $|q q q\rangle+|q q q ; q \bar{q}\rangle$ ) 14. In particular, the non valence term is described by configurations where a nucleon, or a Delta, is coupled to $\pi, \rho$ or $\omega$, with extended coupling constants modeled by phenomenological vertex functions. The momentum dependence of the nucleon and Delta wave functions is chosen according to the Brodsky-Lepage prescription (see, e.g. [15]), while for the mesons a Gaussian Ansatz is adopted. In a frame where $q^{+}=$ $q^{0}+q_{z}=0$, only $j_{\text {em }}^{+}$is necessary, and it is given by a sum of one-body currents for bare baryons
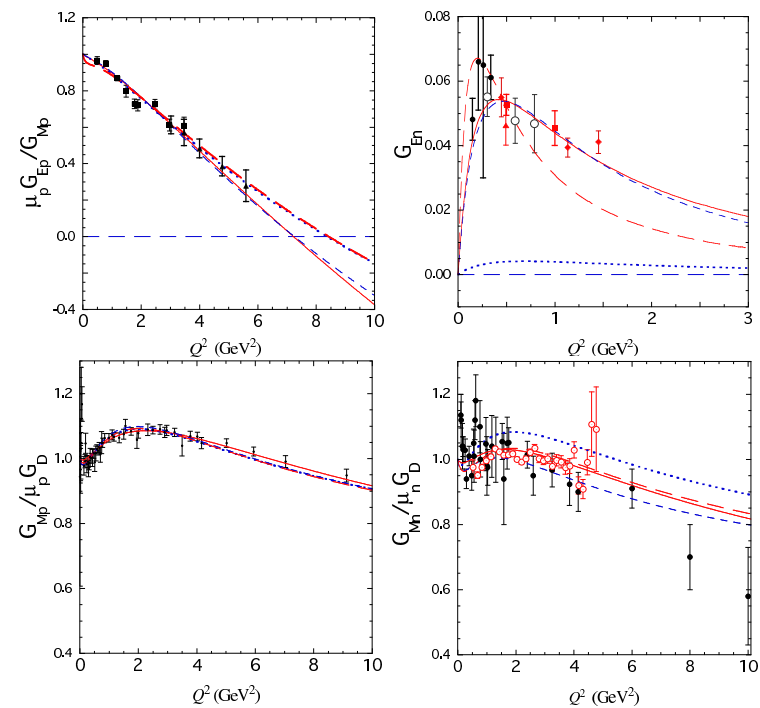

Figure 4. Nucleon ff's, in the SL region, calculated within the manifestly covariant spectator model of Ref. 13. Dotted line: 4 parameters, approximate isospin symmetry in the CQ ff's; shortdashed line: 5 parameters, isospin symmetry fully broken; long-dashed line: 6 parameters, approximate isospin symmetry + two-pion cut; solid line: 9 parameters, isospin symmetry fully broken + two-pion cut. (After Ref. [13])

and mesons. Notably, in the chosen frame, $j_{e m}^{+}$ is diagonal in the Fock space. After determining i) the two parameters in the pion wave function by fitting the corresponding ff (for $\rho$ and $\omega$ the same momentum dependence is assumed) and ii) the cut-off constants in the extended coupling constants, according to the cloudy bagmodel, one obtains the remaining 3 parameters by fitting the theoretical ff's, to 8 experimental values of proton and neutron at low $Q^{2}\left(\mu_{p(n)}\right.$, $G_{A}(0), G_{E}^{n}\left(0.15 \mathrm{GeV}^{2}\right), G_{E(M)}^{p}\left(0.15 \mathrm{GeV}^{2}\right)$ and $\left.G_{E(M)}^{p}\left(0.45 \mathrm{GeV}^{2}\right)\right)$. It is worth noting that with no $S^{\prime}$-wave, one obtains $\mu_{p}=2.87, \mu_{n}=-1.80$, $r_{p}=0.877 \mathrm{fm}$ and $r_{n}^{2}=-0.064 \mathrm{fm}^{2}$. In Ref. [14, a $1 \% S^{\prime}$ component in the Nucleon and Delta wave functions has been also considered, in order to allow a better description of $G_{E}^{n}$. In Fig. 5 , 

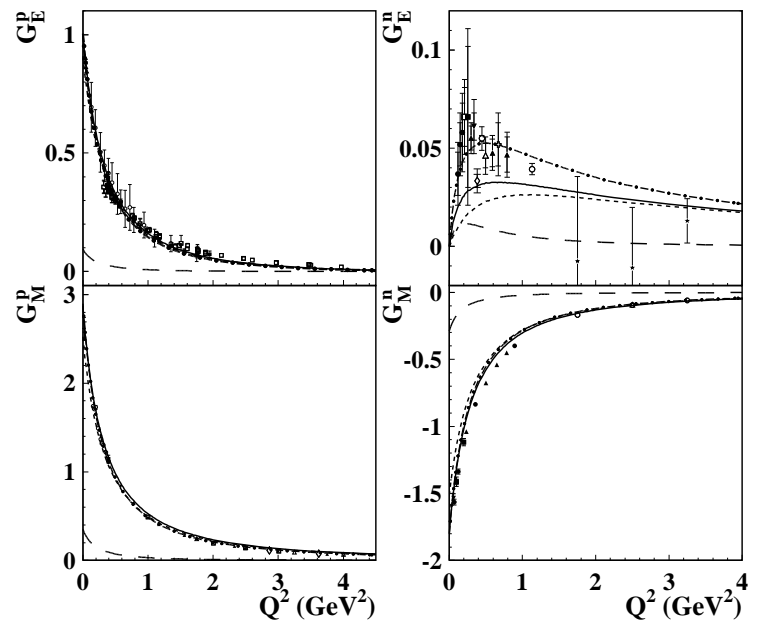

Figure 5. Light-front Nucleon ff's in SL region. Long-dashed curve: meson-cloud contribution; dotted curve: valence-quark contribution; solid curve: the sum of the two contributions; dashdotted curve: the total result with a $1 \% S^{\prime}$-state in (After Ref. [14]).

the results show that the meson cloud is significant only for $Q^{2}<0.5 \mathrm{GeV}^{2}$.

In Ref. [16, a Light-front analysis, already applied to SL and TL Pion ff [17, has been extended to the Nucleon ff's. The starting point is the Mandelstam covariant expression for the matrix elements of the em current. It involves: i) the Dirac propagators of the quarks, ii) the Nucleon Bethe-Salpeter amplitude (BSA), with a proper Dirac structure and a scalar function describing the four-momenta dependence, iii) the quark-photon vertex, to be modeled in order to take into account a microscopical Vector Meson Dominance (VMD) contribution as well [17. After choosing a frame where $q^{+} \neq 0$ (in this frame, $j_{e m}^{+}$is not diagonal in the Fock space), that allows one to deal with the SL and TL regions on the same footing, one can easily integrate on $k^{-}$ (i.e. projecting onto the Light front), if only the Dirac poles are retained. It turns out that the different terms that naively one should expect from physical considerations, can be straightforwardly singled out (see [16). In particular, in the SL region, besides the standard triangle contribution (or valence term), one has a contribution produced by the pair creation (non valence term or Z-diagram term). This term involves both a BSA with two on-shell quarks, that can be related to the valence state, and a BSA with two off-shell quarks, leading to a non-valence term in a Fock expansion of the Nucleon state. In the TL region, there is only the pair contribution. The quarkphoton vertex contains a bare contribution acting in the triangle term (SL only) and a contribution acting when the pair production is present, namely $Z_{b} \times$ a bare term $+Z_{V M D} \times$ a $\mathrm{VMD}$ term, according to the decomposition of the photon state in bare, hadronic (and leptonic) contributions. The momentum dependence of the Nucleon BSA in the valence sector has been approximated by a Nucleon wave function a la BrodskyLepage (i.e., pQCD inspired) [15] with a quark mass $m_{q}=200 \mathrm{MeV}$ and an overall power $7 / 2$. Two parameters are present in the wave function, one is fixed through the anomalous magnetic moments of proton and neutron, $\left(\mu_{t h}^{p}=2.878\right.$, Exp. 2.793, and $\mu_{t h}^{n}=-1.859$, Exp. -1.913), and the other, $p$, that controls the end-point behavior, by the overall fitting procedure (see below). In the non valence sector the momentum dependence is approximated by

$$
\Lambda_{N V}^{S L}=\left[g_{12}\right]^{5 / 2} g_{N \overline{3}} \frac{\left(k_{1}^{+}+k_{2}^{+}\right)}{P_{N}^{\prime+}}\left(\frac{P_{N}^{+}}{k_{\overline{3}}^{+}}\right)^{r}
$$

where $g_{A B}=\left(m_{A} m_{B}\right) /\left[\beta^{2}+M_{0}^{2}(A, B)\right]$ and $r$ is analogous to $p$. A similar expression holds in the TL region. The four adjusted parameters, $Z_{b}^{I S}=Z_{b}^{I V}=Z_{V M}^{I V}, Z_{V M}^{I S}, p$ and $r$, are obtained from the fit of the SL ff's, (even disregarding the proton ratio!) with a $\chi^{2} / D O F \sim 1.7$. The model, illustrated by Figs. 6 and 7, shows that in the SL region the possible zero in $G_{E}^{p}$ is due to the cancellation between the triangle term and the Z-diagram contribution, generated by the higher Fock components of the proton state. Moreover, in the TL region one can see that for the proton some strength is lacking for $q^{2} \sim 4.5$ and $\sim 8(\mathrm{GeV} / \mathrm{c})^{2}$ (as for the Pion [17]), while the neutron remains a challenge. 

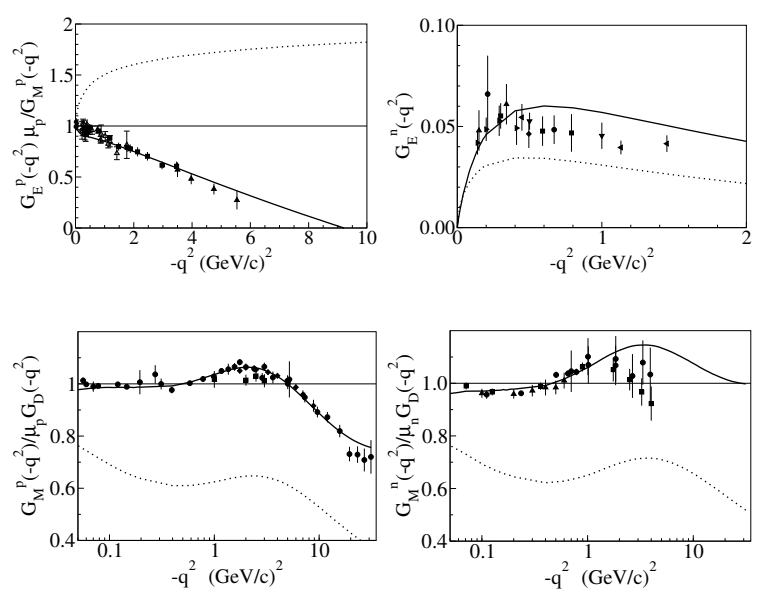

Figure 6. SL Nucleon ff's. Solid line: full calculation. Dotted line: triangle contribution only. $G_{D}=1 /\left(1+\left|q^{2}\right| / 0.71\right)^{2}$. (After Ref. [16])

\section{Conclusions}

Summarizing, Lattice data are becoming more and more accurate, but unfortunately only the SL region can be studied, and for the time being in a limited range of $q^{2}$. Phenomenological models, that can address both SL an TL regions, appear still very appealing, and could contaminate each other, e.g. by supplying the analytic approaches with ingredients microscopically evaluated. In conclusion, one has a set of tools, one fundamental and the others phenomenological, that can positively interact, allowing to gain a more deep insights in the analysis of the Nucleon ff's, that represent an intriguing challenge for the theory, given the highly non perturbative nature of the physical quantities involved in the few-GeV region.

\section{REFERENCES}

1. J. Arrington, C. D. Roberts and J. M. Zanotti, J. Phys. G 34 (2007) S23.

2. C. E. Hyde-Wright and K. de Jager, A. Rev. Nucl. and Part. Sci. 54 (2004)) 217.

3. B. Aubert et al., Phys. Rev. D 73 (2006) 012005 .
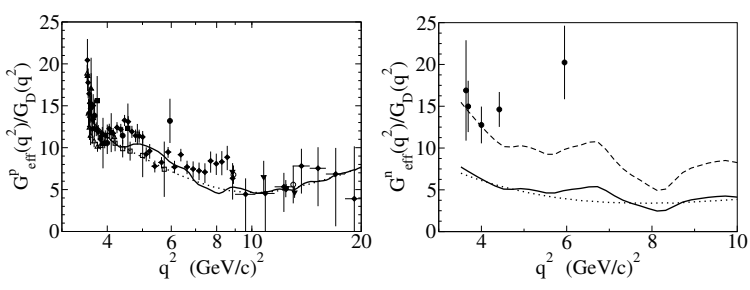

Figure 7. TL Nucleon ff's. Solid line: full calculation. Dotted line: bare contribution only. Dashed line: full calculation $\times 2$ (a guide for the eyes).(After Ref. [16])

4. D. K. Hong, M. Rho, H.-U. Yee and P. Yi, Phys. Rev. D 77 (2008) 014030.

5. R. G. Edwards et al., PoS LAT2006, 121 (2006) 121, hep-lat/0610007.

6. M. Göckeler et al, arXiv:0710.2159 and references quoted therein.

7. C. Alexandrou, G. Koutsou , J. W. Negele and A. Tsapalis, Phys. Rev. D 74 (2006) 034508 .

8. M. A. Belushkin, H.W. Hammer and U.G. Meißner, hep-ph/0608337 and Phys. Rev. C 75 (2007) 035202.

9. S. Pacetti, Eur. Phys. Jou. A 32 (2007) 421.

10. G. Bardin et al., Nucl. Phys. B 411 (1994) 3.

11. R. Baldini et al, Eur. Phys. Jou. C 11 (1999) 709.

12. F. Cardarelli, E. Pace, G. Salmè and S. Simula, Phys. Lett. B 357 (1995) 267.; Nucl. Phys. A 666 (2000) 33c.

13. F. Gross, G. Ramalho, and M. T. Peña, nucl-th/0606029v2 and Phys. Rev. C 77 (2008) 015202.

14. B. Pasquini and S. Boffi, arXiv:0711.0821 and Phys. Rev. D 76 (2007) 074011.

15. S.J. Brodsky, H.C. Pauli, and S.S. Pinsky, Phys. Rep. 301, 299 (1998).

16. J.P.B.C. de Melo, T. Frederico, E. Pace, G. Salmè and S. Pisano, arXiv:0804.1511 and references quoted therein.

17. J.P.B.C. de Melo, T. Frederico, E. Pace, G. Salmè and S. Pisano, Phys. Lett. B 581 (2004) 75, and Phys. Rev. D 73 (2006) 074013 . 